Klaus Robering*

\title{
Kann man die Gebrauchstheorie der Bedeutung in der Semantik gebrauchen?
}

\begin{abstract}
The following article deals with some aspects of special theories of meaning - namely those which consider the meaning of a linguistic sign as a function of its use. Although this approach to meaning is commonly attributed to Wittgenstein, I do not aim at an interpretation or exegesis of this philosopher but rather at a systematic discussion of meaning-as-use-theories. In the first section, I discuss the very notion of a theory of meaning. The next section provides a list of criteria which a theory of meaning should fulfill in order to be a meaning-as-use-theory. The formal architecture of semantic theories is discussed in the third section and - dealing with the simpler case of a referential theory of meaning as an example - the fourth section explains how semantic theories are given a theoretical underpinning by being interpreted within a theory of meaning. The same is done for the case of meaning-as-use-theories in the final section.
\end{abstract}

\section{1. $\quad$ Einleitung}

Die Fragestellungen und Methoden der linguistischen Semantik werden keineswegs allein durch Faktoren bestimmt, die innerhalb der Sprachwissenschaft liegen. Neben den deskriptiven Problemen der Beschreibung des Inhalts bestimmter Wörter und Konstruktionen haben stets auch Entwicklungen in benachbarten Disziplinen - etwa in Semiotik, in Logik und Mathematik, in der Psychologie oder in der Philosophie - für die Semantik eine besondere Rolle gespielt. Ich möchte im folgenden ein spezielles Problem in der Beziehung zwischen Philosophie und Semantik

* PD Dr. Klaus Robering

Institut für Kommunikations- und Softwaretechnik

FLP: Formale Modelle - Logik - Programmierung

Fachbereich 13: Informatik

Technische Universität Berlin

Sekr. FR 6-10

Franklinstr. 28/29

D-10587 Berlin

email:krob@cs.tu-berlin.de 
thematisieren, nämlich die Frage nach der Rolle der sogenannten „Gebrauchstheorie der Bedeutung “ (kurz: Gebrauchstheorie) für die Semantik.

Die Gebrauchstheorie ist ein Beispiel für eine Bedeutungstheorie. In seiner bekannten Einführung in die Sprachphilosophie nennt Alston (1964: ch. 1) als weitere Bedeutungstheorien neben der Gebrauchstheorie noch etwa die Referenztheorie, die Vorstellungstheorie und die behavioristische Verhaltenstheorie der Bedeutung. Bevor ich mich speziell der Gebrauchstheorie der Bedeutung zuwende, werde ich vorab im ersten Abschnitt erklären, was überhaupt eine Bedeutungstheorie ist und welchen Status eine solche Theorie hat. Im zweiten Abschnitt stelle ich sodann eine Liste von Kriterien vor, die eine Theorie erfüllen sollte, wenn sie als Gebrauchstheorie der Bedeutung gelten will. Ausgehend von dieser Liste entwickle ich eine stark vereinfachte Explikation einer Gebrauchstheorie, in der die an Wittgenstein angelehnte Begriffe des „Sprachspiels“" und der „Lebensform“ zentral sind. An einem schon für die Wahrheitsund Referenztheorie herangezogenen Beispiel verdeutliche ich dann, wie ich mir eine gebrauchstheoretisch fundierte Semantik vorstelle.

Im dritten Abschnitt wird erläutert, was ich mir unter einer ,,semantischen Beschreibung“ einer Sprache und unter einer ,,semantischen Theorie" für eine Sprache vorstelle. Im Gegensatz zu den Bedeutungstheorien sind die semantischen Theorien (bestimmter Sprachen) linguistische Theorien. Bedeutungstheorien sind aus der Linguistik herausragende Fundamente für semantische Theorien. An dem einfacheren Beispiel einer Wahrheits- und Referenztheorie der Bedeutung werde ich im vierten Abschnitt zeigen, wie ich mir diese Fundierung vorstelle.

Im fünften Abschnitt kehre ich dann zur Gebrauchstheorie zurück, um zu erläutern, wie eine gebrauchstheoretische Fundierung einer semantischen Theorie aussehen könnte. Die im Titel gestellte Frage ist zum gegenwärtigen Stand der linguistischen Forschung zwar nicht beantwortbar, aber aufgrund der hier diskutierten Beispiele lässt sich ein linguistisches Minimalprogramm entwickeln, dessen Durchführung Voraussetzung für eine Antwort ist ${ }^{1}$.

1 Wie einige Andeutungen in seinen Philosophischen Untersuchungen vermuten lassen, hätte Wittgenstein, der als ein Hauptvertreter der Gebrauchstheorie gilt, die Durchführbarkeit des Minimalprogramms skeptisch beurteilt. Allerdings geht es mir im folgenden auch nicht um eine Wittgensteininterpretation, sondern um eine systematische Erörterung der Möglichkeiten der Gebrauchstheorie. 


\subsection{Bedeutungstheorien}

Die Gebrauchstheorie ist ein Beispiel für eine Bedeutungstheorie; und unter einer Bedeutungstheorie verstehe ich eine Theorie darüber, was Zeichen, speziell sprachliche Zeichen bedeuten. Bedeutungstheorien treffen z. B. Aussagen darüber, worin die Bedeutsamkeit von Zeichen besteht, $o b$ alle Zeichen in derselben Weise bedeutsam sind und welche Typen von Bedeutsamkeit es gibt. Zur Beantwortung solcher Fragen wird sicherlich die empirische Untersuchung des semantischen Systems einzelner Sprachen einiges beizutragen haben. Aber auf rein empirischem Wege sind die bedeutungstheoretischen Probleme nicht lösbar. Bedeutungstheorien gehören nicht zur empirischen Linguistik, sie fundieren diese vielmehr. Eine Bedeutungstheorie enthält starke semiotische und philosophische Elemente ${ }^{2}$. Von Quine stammt das Bild, dass unser Gesamtwissen ein Netz miteinander verknüpfter Annahmen sei: ein „web of belief"; vgl. z. B. Quine/ Ullian (1970). Einige Annahmen - etwa die durch Sinneswahrnehmungen gestützten - befinden sich am Saum des Netzes und befestigen es an der Wirklichkeit; Annahmen theoretischerer Natur befinden sich an zentraleren Stellen des Netzes. Greifen wir dieses Bild auf, so können wir Bedeutungstheorien eher im Zentrum, semantische Theorien (spezieller Sprachen) etwas mehr zur Peripherie hin und semantische Beschreibungen noch weiter zur Peripherie hin lokalisieren.

\subsection{Nicht-korrelationistische Bedeutungstheorien}

Wenn ich gerade etwas umständlich von der „Bedeutsamkeit“ sprachlicher Zeichen statt schlichtweg von deren „Bedeutung“ geredet habe, so sollen damit auch solche Bedeutungstheorien berücksichtigt werden, denen zufolge man eigentlich gar nicht davon reden kann, dass ein Zeichen in dem Sinne etwas bedeute, dass es für ein bestimmtes Objekt steht. Nach einigen Bedeutungstheorien bedeutet etwa das Zeichen Ayers Rock einen bestimmten monolithischen Felsbrocken im Northern Territory Australiens, nach anderen bedeutet dasselbe Zeichen Wahrnehmungs- oder Erinnerungsbilder von Personen, die diesen Ausdruck

$2 \mathrm{Zu}$ Bedeutungstheorien und ihrem Verhältnis zu semantischen Theorien vgl. auch Robering (1997: 85-88). 
benutzen. Nach beiden Arten von Theorien bedeutet das Zeichen Ayers Rock ein Objekt; die Theorien unterscheiden sich lediglich darin, welches Objekt, bzw. von welcher Art die Bedeutung des Zeichens ist. Theorien dieser Art möchte ich als korrelationalistisch bezeichnen, weil sie die Bedeutsamkeit eines Zeichens darin sehen, dass es mit einem bestimmten Objekt - eben seiner Bedeutung - korreliert ist.

Korrelationalistische Positionen werden von der Grammatik des Verbs bedeuten nahe gelegt. Dieses Verb wird im Deutschen transitiv verwendet; und dasselbe gilt für seine Entsprechung in anderen Sprachen. Dies suggeriert, Bedeutsamkeit als eine Beziehung zwischen Zeichen und den Objekten, die sie bedeuten, aufzufassen. Nicht-korrelationalistische Bedeutungstheorien sehen das als einen Fehlschluss an: Nach diesen Theorien besteht die Bedeutsamkeit eines Zeichens eben nicht darin, dass es für ein Objekt - etwa für ein konkretes Ding oder für ein psychisches Gebilde - steht. Dass bedeuten transitiv verwendet wird, spielt hier keine Rolle. Auch das Verb haben wird ja, wenn es nicht als Hilfsverb fungiert, transitiv verwendet werden, und wir schließen von dem Satz (1)

(1) Hans hat einen Hund

ohne weiteres darauf, dass es einen Hund gibt, den Hans besitzt. Aber sollten wir deshalb auch schon von

(2) Hans hat einen Rausch

auf die Existenz eines Rausches schließen, den Hans besitzt? Gibt es also neben betrunkenen Leuten auch noch Räusche, die Leute dann haben, wenn sie betrunken sind? Plausibler als die Annahme von Räuschen als speziellen Objekten neben Betrunkenen ist es, (2) einfach als eine synonyme Umformulierung von

(3) Hans ist betrunken

anzusehen. In ganz analoger Weise deuten nicht-korrelationalistische Theorien Aussagen der Form

(4) Z hat eine Bedeutung

als lediglich alternative und vielleicht etwas irreführende Formulierung von

$Z$ ist bedeutsam (signifikant)

Für eine nicht-korrelationistische Theorie besteht die Bedeutsamkeit eines Zeichens gerade nicht darin, dass es mit einem speziellen Objekt - seiner 
Bedeutung - korreliert ist. Dies charakterisiert solche Theorien aber nur negativ. In ihrer positive Antwort darauf, worin denn die Signifikanz eines Zeichens besteht, unterscheiden sich verschiedene nicht-korrelationalistische Theorien voneinander.

\subsection{Der Status von Bedeutungstheorien}

Ich hatte bereits oben Bedeutungstheorien als eher der Philosophie und Semiotik als der linguistischen Semantik zugehörig bezeichnet. Ich begründe dies damit, dass sie philosophische und tiefliegende semiotische Annahmen über Bedeutungen, über das Meinen und das Verstehen enthalten, die von sprachwissenschaftlichen Argumenten nicht betroffen werden. Linguisten (und andere Wissenschaftler) haben häufig Behauptungen über die spezielle Eigenart oder das Wesen von Bedeutungen aufgestellt. Nach Saussure ist z. B. die Bedeutung eines Zeichens (in seiner Terminologie: eines Ausdrucks) etwas, was er als ,concept“ bezeichnet und der Psychologie zuweist, weshalb seine deutschen Übersetzer „,concept“" mit „Vorstellung“" wiedergeben; vgl. Saussure (1967: 78, 122). Demgegenüber vertritt z. B. Bloomfield eine nicht-korrelationalistische Position, wenn er erklärt:

"The vocal features common to same or partly same utterances are forms; the corresponding stimulus-reaction features are meanings. Thus a form is a recurrent vocal feature which has meaning, and a meaning is a recurrent stimulus-reaction feature which corresponds to a form" (Bloomfield 1926: 27).

Die Bedeutsamkeit eines Zeichens (einer Form) liegt darin, dass sie mit einem Stimulus-Response-Muster verbunden ist: In bestimmten Situationen wird ein Sprecher zur Realisation des Zeichens stimuliert, worauf der Hörer mit einem typischen Response reagiert. Das Zeichen steht natürlich nicht für dieses Stimulus-Response-Muster; es ist aber bedeutsam, weil es mit diesem Verhaltensmuster verknüpft ist.

Wenn ich Bedeutungstheorien dem Bereich der Philosophie/Semiotik zuordne, heißt dies, dass ich nicht glaube, dass sich etwa die Frage, ob Saussure oder ob Bloomfield die richtige Position vertritt, mit sprachwissenschaftlichen Argumenten entscheiden lässt. Die Fragen, worin die Bedeutsamkeit sprachlicher Zeichen besteht und, vorausgesetzt, dass überhaupt eine korrelationistische Position anzunehmen ist, welche Arten von Objekten als Bedeutungen in Frage kommen, stehen außerhalb der 
Linguistik. Darin unterscheiden sich die bedeutungstheoretischen Vorstellungen dieser beiden Autoren etwa von ihren phonologischen.

Es kann durchaus vorkommen, dass die Annahmen einer bestimmten Bedeutungstheorie es als schwierig oder gar aussichtslos erscheinen lassen, eine bestimmte Konstruktion einer natürlichen Sprache semantisch in adäquater Weise zu analysieren. Eine Bedeutungstheorie, die konkrete Objekte als Bedeutungen sprachlicher Zeichen ansieht, hat keinerlei Schwierigkeiten, als Bedeutung von Ayers Rock einen bestimmten Felsbrocken zu identifizieren - aber was soll in einer solchen Theorie das Substantiv Felsbrocken bedeuten? Es müsste für ein konkretes Ding stehen; aber mit Hilfe des Substantivs können wir uns auf viele verschiedene Felsbrocken - auf Ayers Rock, aber auch auf Mt. Conner oder auf einen der Olga Rocks - beziehen. Die skizzierte Bedeutungstheorie hat also bereits Schwierigkeiten mit der Analyse so einfacher prädikativer Konstruktionen wie

(6) Ayers Rock ist ein Felsbrocken

Dies ist eine Schwierigkeit, die die linguistischen Fakten der Bedeutungstheorie bereiten. Aber dass die Theorie in Konflikt mit linguistischen Fakten geraten kann, macht sie noch nicht zu einer linguistischen Theorie. Zunächst sei bemerkt, dass es im Rahmen der skizzierten Theorie nicht angängig ist, auf den geschilderten Einwand zu antworten, Felsbrocken bedeute eben die Menge aller Felsbrocken. Denn im Gegensatz zu einzelnen Felsbrocken ist die Menge aller Felsbrocken kein konkreter (raumzeitlicher) Gegenstand.

Gerade dies zeigt, warum die skizzierte Theorie durch die geschilderte Argumentation von den linguistischen Fakten her jedenfalls in einem gewissen Umfang nicht in Verlegenheit gebracht werden kann. Als philosophiehaltige Theorie wird sie für ihre Annahmen über Bedeutungen philosophische Begründungen geben. Eine Möglichkeit hier wäre etwa ein Verweis auf die Ontologie: Wenn es nur konkrete Dinge (und nichts anderes) gibt, so kommen als Bedeutungen eben nur solche Dinge in Frage. Es ist unwahrscheinlich, dass ein Verfechter dieser Theorie, der seine bedeutungstheoretischen Annahmen so begründet, aufgrund von (6) seine weitreichenden ontologischen Hypothesen revidiert und infolge dessen seine darauf gründende Bedeutungstheorie verwirft. Natürlich bleibt für jeden Vertreter der Theorie die Verpflichtung, eine adäquate Analyse von (6) zu liefern. Linguistische Fakten können eine Bedeu- 
tungstheorie herausfordern; in der Regel können sie sie aber nicht umstoßen. Bedeutungstheoretische Fragen sind in der linguistischen Semantik nicht illegitim, aber sie gehören auch nicht ganz in die Semantik.

\section{Die Gebrauchstheorie der Bedeutung}

Nachdem ich nun eine vorläufige Erklärung vom Status von Bedeutungstheorien und von ihrem Zusammenspiel mit den Semantiken einzelner Sprachen gegeben habe, komme ich nun zur inhaltlichen Charakterisierung der Gebrauchstheorie der Bedeutung.

\subsection{Kennzeichen einer Gebrauchstheorie}

Zunächst ist anzumerken, dass der bestimmte Artikel vor der Bezeichnung Gebrauchstheorie eigentlich unangebracht ist: Es gibt sicherlich mehrere Bedeutungstheorien, die für sich in Anspruch nehmen können, eine Gebrauchstheorie zu sein. Zwar wird der Terminus Gebrauchstheorie fast ausschließlich im Zusammenhang mit den von Wittgenstein in den Philosophischen Untersuchungen (Wittgenstein 1958/69) entwickelten Vorstellungen verwendet ${ }^{3}$, aber Leisi (1952/75: 124) macht in den Nachträgen zu den diversen Auflagen seiner kleinen Einführung in die lexikalische Semantik wiederholt darauf aufmerksam, dass ,die neue [gebrauchstheoretische - KR] Bedeutungsdefinition (von früheren, mehr aphoristischen Bemerkungen abgesehen) zugleich und unabhängig an mehreren Stellen entstanden ist“. Konkret nennt er drei „unabhängig voneinander gemacht[e] Definitionsvorschläge": nämlich neben den Philosophischen Untersuchungen und seiner eigenen Monographie noch Kecskeméti (1952).

Ich will keinen dieser drei Ansätze interpretieren und auch nicht die Dreierliste um weitere Autoren ergänzen. Für die Vorstellungen Wittgensteins sei hier etwa auf von Savignys Kommentar (von Savigny 1994), die Monographie von Hallett (1967) und den Aufsatz von Wiegand

3 Viele Erörterungen der Problematik von Gebrauch und Bedeutung sind lediglich Übungen in Wittgenstein-Exegese, wobei es zumeist um die korrekte Auslegung des berühmten Paragraphen 43 aus dem ersten Teil der Philosophischen Untersuchungen (Wittgenstein 1958) geht. Von einer eher systematisch-theoretischen Perspektive aus untersuchen den Zusammenhang zwischen Gebrauch und Bedeutung die meisten Beiträge in dem Sammelband Meaning and Use (Margalit 1979). 
(1999b) verwiesen, der die Problematik des Zusammenhangs von Bedeutung und Gebrauch bei Wittgenstein speziell von einem linguistischen Standpunkt aus behandelt. Statt eine weitere Wittgenstein-Interpretation zu liefern oder einen der beiden anderen Texte zu untersuchen, will ich einige Eigentümlichkeiten aufzählen, die Gebrauchstheorien gewöhnlich (aber durchaus nicht notwendigerweise) aufweisen, ohne damit etwa behaupten zu wollen, dass z. B. Wittgensteins Vorstellungen durch diese Liste charakterisiert werden.

Gebrauchstheorien der Bedeutung haben normalerweise die folgenden Eigenschaften:

1 Sie sind nicht-korrelationistisch, sehen die Signifikanz von Zeichen also nicht darin, dass ihnen bestimmte Entitäten als ihre Bedeutungen zugeordnet sind. Diese Eigenschaft haben sie aber z. B. mit den behavioristischen Verhaltenstheorien der Bedeutung gemeinsam. Gebrauchs- und Verhaltenstheorien unterscheiden sich hierin etwa von den Bedeutungstheorien Freges und Carnaps, die häufig als Referenztheorien klassifiziert werden.

2 Im Gegensatz zu den behavioristischen Verhaltenstheorien sind Gebrauchstheorien regelorientiert. Das Verhalten, in dem Zeichenbenutzer die Signifikanz von Zeichen zu ihren jeweiligen Zwecken (produktiv oder rezeptiv) ausnutzen, ist regelgeleitet und nicht adäquat als bloßer Response auf einen auslösenden Stimulus zu analysieren.

3 Damit hängt ein weiterer Unterschied zwischen Gebrauchstheorien und behavioristischen Verhaltenstheorien zusammen: Für Gebrauchstheorien ist Signifikanz eine wesentlich soziale Angelegenheit. Das System der Regeln, nach denen signifikante Zeichen verwendet werden, ist sozial basiert. Die Regeln sind Konventionen in der Gemeinschaft der Zeichenbenutzer, sich in bestimmter Weise zu verhalten.

4 Dementsprechend ist Signifikanz eine Eigenschaft von Zeichen, die sich öffentlich manifestiert. Die Bedeutsamkeit eines Zeichens muss sich „ohne Rest“ im Verhalten der kompetenten Zeichenverwender offenbaren.

5 Gebrauchstheorien sind nicht in dem Sinne ,sprachmonistisch“, dass in ihnen allein sprachlicheVerhaltensweisen eine Rolle spielen. Die Signifikanz der in sprachlichen Tätigkeiten verwendeten Zeichen kommt wesentlich durch die Verflechtung dieser Tätigkeiten mit nicht-sprachlichen Aktivitäten in umfassenderen Handlungszusammenhängen zustande.

6 Schließlich haben Gebrauchstheorien typischerweise einen antikompositionalen, holistischen Anstrich. Die Bedeutsamkeit eines komplexen Zeichens wird nicht auf die seiner Teilzeichen und der Art und Weise ihres syntaktischen Aufbaus zurückgeführt. Vielmehr erhalten genau umgekehrt weniger komplexe Zeichen 
ihre Signifikanz dadurch, dass der Gebrauch der umfassenderen Zeichen, als deren Teile sie auftreten, durch Verwendungsregeln festgelegt ist; vgl. den Punkt (2). Diese Verwendungsregeln beziehen sich auf die im Punkt (5) genannten umfassenderen Handlungszusammenhänge.

Dass Gebrauchstheorien nicht-korrelationistisch sind, wird - insbesondere in der älteren Literatur - häufig im Sinne von Occam's Razor als einer ihrer Vorteile angeführt. Durch eine solche Bedeutungstheorie erspare man sich eine Reihe philosophisch dubioser Entitäten. Nun ist viel von dem, was man hier gewöhnlich als besonders einsparenswürdig aufzählt (etwa Sinnesdaten) lediglich von philosophischem oder philosophiegeschichtlichem Interesse. Anderes (etwa Mengen und Eigenschaften) erscheint heute vielen Semantikern und Pragmatikern nicht mehr so entsetzlich wie strengen Empiristen und Nominalisten der 40er und 50er Jahre des 20. Jahrhunderts. Jedenfalls ist im Zusammenhang mit der Nicht-Korrelationalität von Gebrauchstheorien die Frage semantischer Sparsamkeit wohl nicht mehr ausschlaggebend. Diese Eigenschaft der Bedeutungstheorie sieht man heute eher in Verbindung mit dem sozialen Charakter linguistischer Bedeutungen (s. oben den Punkt (3)) und bestimmter verifikationistischer Tendenzen, die sich aus dem obigen Punkt (4) ergeben. In diesem Kontext sei lediglich auf die im Literaturverzeichnis angeführten Arbeiten von Prawitz und Dummett verwiesen.

Der Punkt (2) ist von einiger theoretischer und auch praktischer Bedeutung. Legen Gebrauchstheorien besondere Regelformate für die semantische Charakterisierung von Zeichen fest? In der Logik ist diese Frage insbesondere von Prawitz verfolgt worden; s. Prawitz (1982) und den Bericht (mit weiteren Literaturverweisen) in Robering (1992b). In der Linguistik hat bereits Leisi (1952/75: 16-19) versucht, ein gebrauchstheoretisch motiviertes Regelformat für die semantische Beschreibung zu entwickeln. Er verzichtet jedoch darauf, seine vielfältigen am Englischen und Deutschen angestellten Beobachtungen jeweils genau auf das von ihm vorgeschlagene Format zu bringen. Auf die Frage, wie semantische Regeln formuliert werden können, kommen wir unten in 3.1 zurück.

Die Punkte (3) und (4) hängen eng miteinander zusammen. Zu zeigen, dass es eine im Wesentlichen soziale Angelegenheit ist, (mit einer Äußerung) etwas zu meinen, also eine bestimmte Bedeutung zum Ausdruck zu bringen, ist eines der Hauptanliegen von Wittgensteins Philosophischen Untersuchungen; vgl. in diesem Zusammenhang insbesondere die 
Interpretationen in von Savignys Kommentar (von Savigny 1994). Wenn also, wie auch Quine (1960: ix) sagt, Sprache eine ,soziale Kunstfertigkeit" (social art) ist, dann muss die Bedeutung sprachlicher Zeichen restlos aus dem sozial beobachtbaren Verhalten der Sprachbenutzer hervorgehen. Andernfalls könnte niemand eine Sprache erlernen. Auf diesen Aspekt einer Gebrauchstheorie, auf den insbesondere Dummett immer wieder hingewiesen hat, werden wir im folgenden nicht besonders eingehen, obwohl hier vermutlich die Hauptargumente dafür liegen, eine Gebrauchstheorie der Bedeutung zu akzeptieren; vgl. die Aufsätze in Dummett (1993a). Unsere Fragestellung zielt nicht so sehr auf ,grundsätzliche Pro-Argumente", sondern eher auf das Problem, worin die Leistung einer Gebrauchstheorie für die semantische Beschreibung einer Sprache liegen kann.

Der oben aufgeführte Punkt (6) steht ebenso wie der Punkt (4) in enger Beziehung zu dem Problem des Erwerbs sprachlicher Fähigkeiten. Strenggenommen gibt es nach der etwa in Quine (1960) vertretenen holistischen Bedeutungskonzeption, nach der jedes Element einer Sprache semantisch in Beziehung zu allen anderen steht, zwischen der souveränen Beherrschung einer Sprache und der völligen Unkenntnis keinen weiteren Grad sprachlichen Wissens. Ein gewisser semantischer Holismus wird sicherlich auch von einer jeden Gebrauchstheorie unterstützt: Da die meisten von uns verwendeten Ausdrücke syntaktisch komplex sind, erhalten, wenn der Gebrauch die Bedeutung bestimmt, die Teile der komplexen Ausdrücke ihre Bedeutungen erst durch den Gebrauch der umfassenderen Einheit ${ }^{4}$. Andererseits scheint das Problem des Spracherwerbs eine eher atomistisch-kompositionale Konzeption nahe zu legen, wie sie idealtypisch in der modelltheoretischen Semantik ausgeprägt ist. Dummett versucht hier etwa eine, von ihm als „,molekularistisch“ bezeichnete, vermittelnde Position einzunehmen; vgl. dazu insbesondere auch Tenannt (1987: chs. 5-7). Ein gebrauchstheoretisch motivierter holistischer Ansatz wird exemplarisch für vereinfachte Beispielsprachen von Hamblin (1971) ausgearbeitet, der zeigt, wie satzsemantische Begriffe

4 Plakativ und vereinfachend könnte man sagen: Die Wortsemantik ist eine Funktion der Satzsemantik. 
aufgrund von Wohlgeformtheitsbedingungen für Dialoge definiert werden können ${ }^{5}$.

Im fünften der oben angeführten Punkte geht es darum, dass die sprachlichen Aktivitäten der Mitglieder einer Sprachgemeinschaft in einer semantisch relevanten Weise mit deren nicht-sprachlichen Aktivitäten verknüpft sind. Worauf es hier ankommt, lässt sich gut an den beiden unterschiedlichen Versionen der spieltheoretischen Semantik verdeutlichen, die einerseits von Lorenzen, andererseits von Hintikka entwickelt worden sind ${ }^{6}$. Während die Züge in Lorenzens Dialogspielen ausschließlich sprachliche Aktivitäten sind, handelt es sich bei den semantischen Spielen Hintikkas, wie dieser selbst hervorhebt, um „outdoor games“. So sind etwa bei Hintikka die Quantoren mit nicht-sprachlichen Spielzügen des Suchens und Findens verbunden - ,played in the wide world among whatever objects our statements speak about" (Hintikka 1973: 81).

\subsection{Sprachspiele}

Im Folgenden werde ich aufgrund der in 2.1 vorgelegten Liste einige Vorschläge für den Aufbau einer systematischen Gebrauchstheorie machen. Für eine solche Theorie wäre zunächst die Bestimmung der unter Punkt (5) genannten umfassenderen Handlungszusammenhänge zentral. Einen Terminus von Wittgenstein (1958/69: 7) aufgreifend will ich diese Handlungszusammenhänge als Sprachspiele bezeichnen ${ }^{7}$.

5 Sieht man Dialoge als realisierte Partien spezieller Sprachspiele an, so entspricht das Vorgehen Hamblins unserem Verfahren im 6. Abschnitt. Wir folgen dabei Nowakowska (1973 und 1980), indem wir die Wohlgeformtheitsbedingungen für Sprachspielpartien als durch formale Grammatiken bestimmt ansehen. Eine Sprachspielpartie ist also in diesem Modell ein Wort einer formalen Sprache.

6 Zu Lorenzens Ansatz vgl. etwa die in Lorenzen/Lorenz (1978) gesammelten Aufsätze sowie (Rückert (2001) für neuere Entwicklungen; zu Hintikkas spieltheoretischer Semantik siehe z. B. Hintikka/ Kulas (1983) und zu Hintikkas Wittgensteininterpretation Hintikka (1996 und 2000).

7 Es sei nochmals darauf hingewiesen, dass es mir auf eine Wittgenstein-Interpretation nicht ankommt. Ich will also insbesondere nicht behaupten, mit dem oben Folgenden eine korrekte Explikation des Wittgensteinschen Sprachspielbegriffs geliefert zu haben. Wittgensteins Ausführungen zum Konzept der „Familienähnlichkeit“" in den Philosophischen Untersuchungen, das er ja gerade am Spielbegriff exemplifiziert, legen sogar nahe, dass er selbst eine systematische, in einer Definition gipfelnde Explikation des Sprachspielbegriffs nicht für möglich gehalten hätte. Hier und im Folgenden benutze ich die Philosophischen Untersuchungen lediglich als Anregung. 
Wittgenstein führt zur Verdeutlichung seiner Argumentation im Anfangsteil der Philosophischen Untersuchungen mehrere einfache Beispiele von Sprachspielen an; vgl. etwa die $\S \S 1$ (letzter Absatz), 2, 7, 8, 15, 41, 48 in Wittgenstein (1958/69). Zu einem Sprachspiel $\boldsymbol{G}$ gehört zunächst eine Menge $P$ von Mitspielern. Das in $\S 48$ von Wittgenstein beschriebene Spiel ist ein Solitärspiel; Sprachspiele mit nur einem Mitspieler sind also zugelassen. Das ist aber auch die unterste Grenze für die Spielerzahl; $P$ muss also zumindest einen Mitspieler enthalten.

Als nächstes haben wir die Spielzüge von $\boldsymbol{G}$ festzulegen. Jeder Spielzug gehört einer von zwei Arten an: Er besteht entweder in einer Äußerungsaktivität oder in einer nicht-semiotischen Tätigkeit. Im Sprachspiel des $§ 2$ der Philosophischen Untersuchungen äußert z. B. ein Bauender einen der Ausdrücke Würfel, Säule, Platte oder Balken und sein Gehilfe schafft daraufhin einen entsprechenden Baustein herbei. Im ersten Sprachspiel des $\S 7$ weist der Lehrer auf einen Gegenstand, woraufhin der Schüler diesen Gegenstand mit dem passenden Ausdruck benennt ${ }^{8}$.

Zur Charakterisierung der Äußerungsaktivitäten haben wir festzulegen, was die äußerbaren Ausdrücke sind und welche innere Struktur sie haben. $\mathrm{Zu}$ einem Sprachspiel $\boldsymbol{G}$ gehört also jeweils eine Sprache $L$, wobei nicht unbedingt gefordert ist, dass jeder Ausdruck von $L$ (oder auch nur jede Aussage, jede Frage usw.) tatsächlich auch in $\boldsymbol{G}$ verwendet werden kann. Durch die Angabe des geäußerten Ausdrucks ist eine Äußerung allerdings noch nicht hinreichend charakterisiert: Es fehlt neben der Angabe des Sprechers und des Adressaten noch die spezifische illokutionäre Kraft, mit der der Ausdruck geäußert wird. Im Sprachspiel des $\S 2$ handelt es sich bei den Äußerungen durchweg um Befehle. Im $\S 7$ lassen sich jedoch die Äußerungen des Schülers zwanglos als Behauptungen (Dies ist eine Platte usw.) interpretieren. Zur Angabe $L$ der Sprache gehört also auch noch die Angabe $F$ einer Menge von Kräften ${ }^{9}$.

8 Das zweite Sprachspiel des $§ 7$ - die ,,noch einfachere Übung“(Wittgenstein 1958: 292) - besteht darin, dass der Lehrer ein Ausdruck äußert und der Schüler diesen dann nachzubilden versucht.

9 Der auf Frege zurückgehenden Unterscheidung zwischen illokutionärer Kraft und propositionalem Gehalt einer Äußerung, die in der Sprechakttheorie aufgegriffen und systematisiert wird, steht Wittgenstein äußerst kritisch gegenüber; vgl. Wittgenstein (1958/ 69: $§ \S 22-24)$; siehe dazu auch Dummetts Kommentar in Dummett (1991b: 239). 
Unter einem Äußerungszug von $\boldsymbol{G}$ wollen wir ein Quadrupel ( $m, \varphi$, $f, n)$ verstehen, bei dem $m$ und $n$ Mitspieler, $\varphi$ ein Ausdruck der Sprache $L$ und $f$ eine illokutionäre Kraft ist: Der Sprecher $m$ äußert den Ausdruck $\varphi$ mit der illokutionären Kraft $f$ gegenüber dem Adressaten $n$. Ein NichtÄußerungszug soll ein Paar $(m, t)$ sein, in dem $m$ wiederum ein Mitspieler und $t$ ein Element einer für $\boldsymbol{G}$ charakteristischen Klasse $T$ von Handlungsweisen ist.

Die Regeln (vgl. den Punkt (2) in der Liste im Abschnitt 2.1) sind in den von Wittgenstein beschriebenen Sprachspielen im wesentlichen Abfolgeregeln. Auf einen bestimmten Zug $z_{0}$ eines Mitspielers können aus der Gesamtheit aller möglichen Züge nur ganz bestimmte Gegenzüge $z_{1}$, $z_{2}, z_{3}, \ldots$ folgen, während andere ausgeschlossen sind. Ein anderer Zug würde gegen die Regeln von $\boldsymbol{G}$ verstoßen. Die Relation, die zwischen einem Zug und einem von ihm erlaubten Folgezug besteht, will ich mit $\Rightarrow$ bezeichnen. In der gerade erwogenen Situation gilt also: $z_{0} \Rightarrow z_{1}, z_{0} \Rightarrow z_{2}$, $z_{1} \Rightarrow z_{3}$ usw. Damit das Spiel überhaupt beginnen kann, hat man aus der Gesamtheit der Züge die Teilklasse der initialen Züge auszuzeichnen. Ebenso sind finale Züge als Endpunkte des Spiels zu bestimmen.

Damit haben wir nun alle Bestimmungsstuicke von $\boldsymbol{G}$ zusammen. Ein Sprachspiel $\boldsymbol{G}$ soll ein 7-Tupel $(P, L, F, T, \Rightarrow, I, E)$ sein: $P$ ist die Gesamtheit der Mitspieler, $L$ die von ihnen benutzte Sprache, $F$ die Gesamtheit der illokutionären Kräfte, unter denen sie Ausdrücke von dieser Sprache äußern, $T$ die Typen nicht-linguistischer Tätigkeiten, die sie vollziehen, $\Rightarrow$ ist die Abfolgerelation zwischen Zügen und $I$ bzw. $E$ ist schließlich die Klasse der Anfangs- bzw. die der Endzüge von $\boldsymbol{G}$. Ein Zug ist entweder ein Äußerungszug oder ein Nicht-Äußerungszug. Die Gesamtheit der Äußerungszüge von $\boldsymbol{G}$ soll $\mathbf{U Z}(G)$, die der Nicht-Äußerungszüge $\mathbf{N Z}(\boldsymbol{G})$ sein. Wir hatten bereits festgelegt, dass $\mathbf{N Z}(\boldsymbol{G}) \subseteq P \times T$. Ist $\mathbf{U t t}(\mathrm{L})$ die Menge der in Sprachspielen äußerbaren Ausdrücke von $L$, so ist $\mathbf{U Z}(\boldsymbol{G})$ $\subseteq P \times \mathbf{U t t}(\mathrm{L}) \times F \times P$.

Eine Partie des Sprachspiels $\boldsymbol{G}=(P, L, F, T, \Rightarrow, I, E)$ ist eine Abfolge $\mathrm{p}=\left(z_{0}, z_{1}, \ldots, z_{n}\right)$ von Zügen, die folgenden drei Bedingungen genügt: (1.) $z_{0} \in I ; z_{0}$ muss ein Anfangszug von $\boldsymbol{G}$ sein. (2.) Für $m<n$ gilt jeweils $\mathrm{z}_{m} \Rightarrow z_{m+1}$; auf einen vorgegebenen Zug folgt stets ein im Sinne der Regeln von $\boldsymbol{G}$ erlaubter Zug. (3.) $\mathrm{z}_{n} \in E$; der letzte Zug muss ein Endzug des Spiels sein. 


\subsection{Beispiel: Wittgensteins einfaches Sprachspiel}

Wir können nun das von Wittgenstein (1958/69: § 2) beschriebene Sprachspiel leicht auf die Form bringen, die unsere Definition des Sprachspielbegriffs verlangt. Dieses Spiel wird von zwei Parteien gespielt: $P=$ \{Bauender, Gehilfe\}. Die Sprache $L$ des Spiels besteht lediglich aus den vier Ausdrücken Würfel, Säule, Platte und Balken; wir nennen diese Sprache im Folgenden die „Minimalsprache“ ML. Die vier Ausdrücke von ML werden ausschließlich im auffordernden Modus verwandt: $F=$ $\{!\}$. Es gibt vier Typen nicht-linguistischer Tätigkeiten: das Herbeibringen (h) eines Würfels (w), einer Säule (s), einer Platte (p) und eines Balkens (b). Es ist also $T=\{\mathrm{hw}, \mathrm{hs}, \mathrm{hp}, \mathrm{hb}\}$.

Die für das Spiel charakteristische Sequenzregel lässt sich in zwei Teilregeln zerlegen: (1.) Auf einen Äußerungszug der Form (Bauender, $\varphi, !$, Gehilfe) muss stets ein Nicht-Äußerungszug (Gehilfe, $h \varphi$ ) folgen. Man hat also Abfolgen der Gestalt (Bauender, $\varphi, !$, Gehilfe) $\Rightarrow$ (Gehilfe, $h \varphi$ ), wobei natürlich für $\varphi=$ Würfel gelten soll, dass $h \varphi=h w$ usw. (2.) Auf einen Zug $z$ des Gehilfen darf stets eine erneute Aufforderung (Bauender, $\psi$, !, Gehilfe) des Bauenden erfolgen: $z \Rightarrow$ (Bauender, $\psi$, !, Gehilfe).

Anfangszüge des Spiels sind Aufforderungen (Bauender, $\varphi, !$, Gehilfe) des Bauenden, Endzüge Herbeischaffungen (Gehilfe, $h \varphi$ ) des Gehilfen. Eine Partie dieses Spiels besteht damit aus endlich vielen Aufforderungen des Bauenden, denen jeweils entsprechende Akte des Herbeischaffens durch den Gehilfen folgen. Um auf dieses Spiel leichter Bezug nehmen zu können, wollen wir es im folgenden $\mathbf{W}$ oder auch das $\mathbf{W}$-Spiel nennen.

\subsection{Lebensformen}

$\mathrm{Zu}$ einer Sprache gehören normalerweise mehrere Sprachspiele. Wittgenstein beschreibt z. B. für die fiktive Sprachgemeinschaft, die sich der Minimalsprache ML bedient, neben dem W-Spiel noch zwei weitere Sprachspiele, in denen der Gebrauch von ML gelehrt wird; vgl. Wittgenstein (1958/69: § 7).

Beide Lehrspiele sind Zweipersonenspiele zwischen einem Lehrer und einem Schüler. Im zweiten Spiel, das Wittgenstein als , die noch einfachere Übung " bezeichnet, spricht der Lehrer jeweils einen der vier Ausdrücke von ML aus, woraufhin der Schüler die Äußerung des Lehrer nachzuahmen hat. In diesem Spiel soll der Schüler die Phonologie der Mini- 
malsprache zu beherrschen lernen. In dem von Wittgenstein im $§ 7$ zuerst genannten Spiel weist der Lehrer auf ein Bauelement, woraufhin der Schüler dieses zu benennen hat. Hier geht es also um den Erwerb semantischen Wissens, worauf sich die vier Ausdrücke der Minimalsprache beziehen. Man kann leicht Beschreibungen dieser beiden Spiele geben, die dem im Abschnitt 2.2 angegebenen Standardformat entsprechen.

Der Witz der beiden Lehrspiele besteht natürlich darin, die Schüler auf eine Teilnahme an dem Bauspiel vorzubereiten. Personen, die die Rolle des Schülers im Lehrspiel innehaben, sollen später beim Bauen erfolgreich als Bauende oder Gehilfen auftreten. Die verschiedenen Spiele hängen also über ihre jeweiligen Spielermengen miteinander zusammen. Eine maximale Gesamtheit $\boldsymbol{L}$ miteinander zusammenhängender Sprachspiele $\boldsymbol{G}_{1}, \boldsymbol{G}_{2}, \ldots$ will ich in Anlehnung an Wittgenstein (1958/69: $\S \S 19$, 23) als Lebensform bezeichnen: $\boldsymbol{L}=\left\{\boldsymbol{G}_{1}, \boldsymbol{G}_{2}, \ldots\right\}$. Der Zusammenhang der Sprachspiele besteht zum einen darin, dass sie sämtlich dieselbe Sprache $L$ betreffen. Zum anderen müssen die Mitspieler der zu einer Lebensform zusammengefassten Sprachspiele in einem bestimmten Verhältnis zueinander stehen. Wittgensteins Beispiel beruht, wie wir gesehen haben, auf der Vorstellung, dass der Bauende und sein Gehilfe einmal in der Rolle des Schülers an den Sprachlehrspielen teilgenommen haben. Ferner konstituiert sich der Zusammenhang zwischen den Spielen einer Lebensform in der Gesamtheit der in ihnen vorkommenden illokutionären Kräfte. Diese Kräfte sind ja in bestimmten Praktiken der Sprachgemeinschaft verankert, zu denen wiederum bestimmte Sprachspiele gehören. Zur direktiven Kraft gehören etwa die Praktiken des Bestrafens, Tadelns, Lobens und Auszeichnens mit entsprechenden Sprachspielen ${ }^{10}$.

\section{Semantik}

Unsere Titelfrage betrifft das Verhältnis zwischen der Semantik (einer Einzelsprache) und der Gebrauchstheorie der Bedeutung. Nachdem wir den Begriff einer Gebrauchstheorie der Bedeutung näher geklärt haben,

10 Der Zusammenhang zwischen den verschiedenen Sprachspielen einer Lebensform gleicht der Art und Weise, wie in der sog. ,,strukturalistischen“ Wissenschaftstheorie die unterschiedlichen potentiellen Modelle einer empirischen Theorie durch „Querverbindungen“ (,,internal links“, ,constraints“) miteinander verbunden gedacht werden; vgl. Balzer/ Moulines/ Sneed (1987: 40-47). 
müssen wir uns nun der Semantik zuwenden. Da wir bedeutungstheoretische Fragen aus der Semantik ausgeklammert haben, stellt sich jetzt dringend die Frage, womit sich die Semantik eigentlich überhaupt beschäftigt. Semantik wird ja üblicherweise als die Lehre von der Bedeutung der Zeichen verstanden und als solche müsste sie doch zuallererst klären, worin Bedeutung besteht. Nun, positiv gewendet, bedeutet die Trennung semantischer und bedeutungstheoretischer Fragen, dass die Semantik mit einer jeden Bedeutungstheorie, insbesondere also auch mit einer nicht-korrelationistischen Bedeutungstheorie verträglich sein muss. Dann spielt aber der korrelationistische Begriff der Bedeutung - als etwas, was einem Zeichen zugeordnet ist - meines Erachtens selbst gar keine Rolle in der Semantik.

\subsection{Urteile und Regeln}

Dies mag paradox und exzentrisch klingen, ist aber eigentlich eine sehr traditionelle Position. Strukturalistischen Vorstellungen zufolge hat es die Linguistik ja gar nicht mit den substanziellen Bestandteilen des Sprachsystems zu tun, sondern mit den Beziehungen innerhalb dieses Systems. Für die Semantik heißt dies, dass die semantischen Beziehungen - und nicht deren Relata - der eigentliche Gegenstand dieser Disziplin sind; vgl. etwa Lyons (1977: ch. 9). Beispiele semantischer Beziehungen sind etwa: Folge und Äquivalenz, Kontrarietät und Kontradiktionalität sowie Hyponymie und Hyperonymie. Semantische Eigenschaften sind nichts weiter als einstellige semantische Beziehungen; zu den semantischen Beziehungen treten also auch noch Wahrheit, Falschheit und Indefinitheit (Wahrheitswertlosigkeit), die Eigenschaften, tautologisch und kontradiktorisch zu sein usw.

Im folgenden will ich Zeichen, für welche es sinnvoll ist, die Frage nach Wahrheit oder Falschheit zu erheben, Aussagen nennen. Die Folgebeziehung ist eine Relation zwischen einer Menge von Aussagen (den Annahmen) und einer einzelnen Aussage (der Konklusion): Man sagt, die Konklusion $\varphi$ folge aus der Menge $\Phi$ der Annahmen. Hierfür will ich $\Phi<\varphi$ schreiben. Im Spezialfall kann $\Phi$ eine Einermenge $\Phi=\{\psi\}$ sein. In diesem Fall schreiben wir $\psi<\varphi$ statt des pedantischeren $\{\psi\}<$ $\varphi$.

Semantische Beziehungen sind, wie bereits aus der obigen Liste hervorgeht, keineswegs auf Aussagen beschränkt. Die Wörter Pferd und 
Tier sind natürlich (im Deutschen) keine Aussagen; zwischen ihnen besteht aber die semantische Relation der Hyponymie. Ein anderes Beispiel für eine semantische Beziehung, an der Nicht-Aussagen beteiligt sind, ist die oben noch nicht erwähnte Antwort-Frage-Beziehung. Ich schreibe $\varphi-$ ? $\alpha$, wenn $\mathrm{j}$ eine Antwort auf die Frage $\alpha$ ist. Hier ist $\alpha$ eine Frage, also jedenfalls keine Aussage; $\varphi$ kann eine Aussage sein. Ob es auch eine Aussage sein muss, ob Antworten, die nicht Aussagen sind, stets elliptische Kurzformen für Antworten sind, ist ein Streitpunkt in der Semantik der Fragen.

Formeln wie $\Phi<\varphi$ oder $\varphi-$ ? $\alpha$, in denen das Bestehen semantischer Beziehungen zwischen schematisch angedeuteten Argumenten ausgesagt wird, bezeichne ich als Urteile. Weitere Beispiele für Urteile sind etwa $\varphi:$ T ( $\varphi$ ist wahr) und $\varphi:$ F ( $\varphi$ ist falsch). Semantische Regeln stellen Beziehungen zwischen Urteilen her. Ich formuliere sie mit Hilfe der Pfeilnotation so: $M \Rightarrow N$. Dies soll bedeuten, dass zumindest eines der Urteile aus $N$ stimmt, falls dies für alle Urteile aus $M$ zutrifft. $M$ ist das Antezedens, $N$ das Sukzedens der Regel.

Semantische Regeln sind natürlich für das jeweils untersuchte Sprachsystem spezifisch. Für das Deutsche wären etwa Regeln wie

(7a) ( $\zeta$ ist Maurer) $: \mathrm{T} \Rightarrow(\zeta$ ist Handwerker) $: \mathrm{T}$

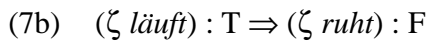

(7c) ( $($ ist Vater von $\xi): \mathrm{T},(\xi$ ist Vater von $\chi): \mathrm{T} \Rightarrow(\zeta$ ist Großvater von $\chi): \mathrm{T}$

denkbar. Die beiden Regeln (7a) und (7b) haben jeweils nur eingliederigere Antezedentien und Sukzedentien. Ein etwas komplexeres Beispiel ist bereits (7c) mit seinem zweigliederigen Antezedens. Ein Beispiel für eine Regel mit einem zweigliederigen Sukzedens wäre etwa

(8) ( $\zeta$ und $\xi$ sind Geschwister) : $\mathrm{T} \Rightarrow(\zeta$ ist Bruder von $\xi): \mathrm{T},(\zeta$ ist Schwester von $\xi): \mathrm{T}$

Eine Regel \{\}$\Rightarrow M$ mit leerem Antezedens besagt, dass zumindest ein Urteil des Sukzedens $M$ richtig sein muss. Ist $M$ also eine Einermenge $\{A\}$, so wird mit \{\}$\Rightarrow\{\mathrm{A}\}$, wofür wir einfacher \{\}$\Rightarrow A$ schreiben wollen, gesagt, dass das Urteil $A$ stimmt. Eine solch ausgeartete semantische Regel könnten wir als ,sachverhaltskonstatierend“ bezeichnen: Sie stellt den semantischen Sachverhalt fest, der im Urteil $A$ beschrieben wird. Umgekehrt sagt eine Regel $M \Rightarrow\{\}$ mit leerem Sukzedens, dass 
nicht alle Urteile des Antezedens $M$ stimmen können. Würden nämlich alle diese Urteile stimmen, müsste es ja erklärungsgemäß im Sukzedens auch ein wahres Urteil geben. Das Sukzedens ist hier aber leer.

Eine semantische Beschreibung einer Sprache $L$ stelle ich mir folgendermaßen vor: (1.) Zunächst müsste eine erschöpfende Liste der für $L$ relevanten semantischen Beziehungen angegeben werden. Durch diese Liste wird die Gesamtheit der für $L$ relevanten Urteile und die Klasse der für diese Sprache überhaupt in Frage kommenden Regeln festgelegt. Diese Gesamtheit bezeichne ich mit $\operatorname{ReR}(L)$. Nicht jede Regel aus $\operatorname{ReR}(L)$ stimmt für $L$. Es muss daher (2.) aus der Gesamtheit der möglichen die Teilgesamtheit der für $L$ stimmenden Regeln ausgegrenzt werden. Die Teilklasse der für $L$ stimmenden Regeln soll $\operatorname{KoR}(L)$ heißen. Wenn eine Regel aus $\operatorname{ReR}(L)$ als nicht auf $L$ zutreffend erwiesen werden kann, so stimmt sie für diese Sprache nicht; $\mathbf{I n R}(L)$ soll die Gesamtheit der für $L$ nicht-stimmenden Regeln sein. Die beiden Klassen $\operatorname{KoR}(L)$ und $\operatorname{InR}(L)$ sind disjunkte Teilklassen von $\mathbf{R e R}(L)$. Sie müssen aber zusammen nicht unbedingt $\operatorname{ReR}(L)$ erschöpfen.

\subsection{Beispiel: Die Minimalsprache ML im W-Spiel}

Um die eingeführten Begriffe zu erläutern, wollen wir die Minimalsprache ML betrachten, derer sich die Teilnehmer des $\mathbf{W}$-Spiels bedienen und die Wittgenstein im $\S 2$ seiner Philosophischen Untersuchungen beschreibt, s. Wittgenstein (1958/69: 290). Diese besteht aus nur vier Ausdrücken: Würfel, Säule, Platte und Balken und dient der Verständigung über die mit ihnen bezeichneten Bauelemente. Obwohl er das nicht ausdrücklich sagt, suggeriert Wittgensteins Darstellung, dass außer Würfeln, Säulen, Platten und Balken keine anderen Bauelemente vorhanden sind und das jedes Bauelement zu genau einer der vier Klassen gehört ${ }^{11}$.

Obwohl die angeführten Ausdrücke von dem Bauenden als Befehle gebraucht werden, lassen sie sich auch zwanglos als Aussagen deuten ${ }^{12}$.

11 Wittgenstein diskutiert jedenfalls nicht die Möglichkeit, dass z. B. die Klassifikation eines Bauelements als Säule oder Platte von seiner Lage (vertikal bzw. horizontal) abhängig sein könnte.

12 Dies ist gerade die Eigenschaft dieses Beispiels, auf die es Wittgenstein für seine Argumentation besonders ankommt. Ich verstehe hier Wittgenstein so, dass selbst dort, wo eine solche Auffassungsweise und eine auf ihr gründende referenztheoretische Analyse des Sprachgebrauchs möglich ist, diese doch immer noch nicht erschöpfend ist. 
Wir können daher als Urteile in der Semantik von ML Wahrheits- und Falschheitszuschreibungen wie: Platte: T, Säule : F usw. verwenden. In unserer extrem einfachen Sprache haben wir anders als in den Regelbeispielen (7) und (8) keinerlei Veranlassung, objektsprachliche Ausdrücke zum Zwecke der Generalisierung schematisch anzudeuten, wozu in (7) und (8) die Buchstaben $\zeta$ und $\xi$ dienen. Damit ist die Anzahl der für unsere Minimalsprache relevanten Urteile auf 8 beschränkt. Aus 8 Urteilen lassen sich $2^{8}$, also 256 Urteilsklassen bilden. Da in jeder Regel 2 Urteilsklassen vorkommen, erhält man somit für $\operatorname{Re} \operatorname{Re}(\mathrm{ML})$ die doch schon beträchtliche Zahl von $256^{2}$, also von 65.536 relevanten Regeln.

Dass alle Bauteile, auf die die Kommunikation zwischen dem Bauenden und seinem Gehilfen abzielen, Würfel, Säulen, Platten oder Balken sind, wird in der folgenden semantischen Regel gesagt:

$$
\{\} \Rightarrow \text { Würfel : T, Säule : T, Platte : T, Balken : T. }
$$

Dass sich die vier Ausdrücke gegenseitig ausschließen, beinhalten in ihrer Gesamtheit die semantischen Regeln, die das folgende Schema instanziieren:

(10) $\varphi: T \Rightarrow \psi:$ F für $\varphi \neq \psi$.

Eine konkrete Instanz für das Regelschema (10) ist etwa:

(11) Würfel : $\mathrm{T} \Rightarrow$ Platte : F.

Die Regel (9) und jede Instanz der Regel (10) stimmen für unsere Minimalsprache; sie gehören also zu KoR(ML). Die Regel Würfel : $\mathrm{T} \Rightarrow$ Platte : T ist hingegen nicht korrekt, sie gehört zu InR(ML). Die sachverhaltskonstatierende Regel \{\}$\Rightarrow$ Würfel : F kann man sinnvollerweise aber weder als stimmend, noch als nicht-stimmend klassifizieren.

\subsection{Regelwerke}

Die bekannte Terminologie Chomskys aufgreifend, könnte man sagen, dass man, wenn man für eine Sprache $L$ die beiden Regelgesamtheiten $\operatorname{KoR}(L)$ und $\mathbf{I n R}(L)$ angegeben hat, damit für die Semantik die Ebene der Beobachtungsadäquatheit erreicht hat. Der Einblick in die Semantik von $L$ ist damit noch nicht sehr tief. Bloße Auflistung der für $L$ stimmenden und nicht-stimmenden Regeln sind auch nicht sehr erhellend, da sie den systematischen Zusammenhang zwischen den Regeln nicht wiedergeben. Einige stimmende Regeln werden sich ja z. B. aus anderen ergeben. 
Ja, es ist sogar zweifelhaft, ob man überhaupt die für $L$ stimmenden Regeln unabhängig von den zwischen ihnen bestehenden Zusammenhängen angeben kann. Gibt es unendlich viele solcher Regeln, so ist kaum zu sehen, wie dies möglich sein soll.

In einem solchen Fall könnte man immer noch hoffen, dass es unter den Regeln von $\mathbf{K o R}(L)$ einige besonders fundamentale gibt, die in ihrer Gesamtheit effektiv angebbar sind und aus denen man alle anderen Regeln aus $\mathbf{K o R}(L)$ mit Hilfe endlich vieler Schlussverfahren gewinnen kann. Solche Schlussverfahren sind selbst wieder Regeln; gegenüber den semantischen Regeln haben sie den Status von Metaregeln. Eine Gesamtheit solcher Schlussverfahren will ich als ein Regelwerk bezeichnen. Ein aufgrund der Erklärungen zum Regelpfeil naheliegendes Regelwerk ist etwa das folgende; vgl. Scott (1971: 796):

(12a)

$$
\overline{M \Rightarrow N} \text {, falls } M \cap N \neq\{\}
$$

$$
\frac{M \Rightarrow N}{M^{\prime} \Rightarrow N^{\prime}}, \text { falls } M^{\prime} \supseteq M \text { und } N^{\prime} \supseteq N
$$

(12c)

$$
\frac{M \Rightarrow N, A \quad A, M \Rightarrow N}{M \Rightarrow N}
$$

Die drei in (12) aufgeführten Schlussregeln stellen in ihrer Gesamtheit eine alternative Formulierung der sogenannten ,strukturellen Regeln“ des Sequenzenkalküls für die klassische Logik dar; vgl. Gentzen (1934/ 1935: 192) ${ }^{13}$. In unserem Kontext können wir die „Strukturalität“ der angeführten Schlussregeln so verstehen, dass sie ganz generell von beliebigen Urteilen (bzw. Urteilsmengen) gelten. In der Metaregel (T) wird ja z. B. nicht verlangt, dass im Urteil $A$ etwa von einer Folge- oder einer Antwort-Frage-Beziehung die Rede ist.

Die Reflexivitätsregel (R) von (12a) erlaubt die Annahme trivialer Regeln, bei denen zumindest ein Antezedensglied auch als Sukzedensglied auftritt. Stimmen alle Antezedensglieder, so stimmt insbesondere

13 Zwischen Gentzens logischen Theorien und der Gebrauchstheorie der Bedeutung besteht ein enger Zusammenhang, über den ich in Robering (1992b) berichte. 
auch das Antezedens und Sukzedens gemeinsame Glied. Trivialerweise stimmt dann also auch ein Sukzedensglied. Die Monotonieregel (M) von (12b) gestattet es, in das Antezedens und in das Sukzedens einer semantischen Regel redundante Elemente einzubringen. Stimmt jedes Urteil aus $M^{\prime}$, so muss auch insbesondere jedes Urteil der Teilmenge $M \subseteq M^{\prime}$ stimmen. Nach der Voraussetzung $M \Rightarrow N$ ist dann zumindest ein Urteil von $N$ korrekt, weshalb auch eines der Obermenge $N^{\prime} \supseteq N$ stimmt. Die Transitivitätsregel (T) ist eine Form des Kettenschlusses. Sei angenommen, dass alle Urteile aus $M$ stimmen. Wegen $M \Rightarrow N, A$ ist dann auch ein Urteil aus $N$ (Fall 1) oder aber das Urteil $A$ (Fall 2) korrekt. Im ersten Fall hat man bereits $M \Rightarrow N$. Im zweiten Fall erhält man aus $A, M \Rightarrow N$, dass ein Urteil aus $N$ stimmen muss. Es gilt also auch hier $M \Rightarrow N$.

Eine semantische Theorie $\Theta$ - kurz: eine Semantik - für die Sprache $L$ stelle ich mir nun (annäherungsweise) als aus zwei Komponenten bestehend vor: (1.) einem Regelkern $\mathbf{K}(\Theta)$ und (2.) einem Regelwerk $\mathbf{R w}(\Theta)$. Der Regelkern ist eine entscheidbare Teilklasse von $\operatorname{Re}(L)$, das Regelwerk eine endliche Menge konstruktiver Metaregeln, wie sie im Beispiel (12) veranschaulicht werden. Die Theorie $\Theta$ ist korrekt für $L$, wenn keine Regel, die in dieser Theorie aus dem Kern durch endlichmalige Anwendung von Regeln des Regelwerks gewonnen werden kann, für $L$ nicht stimmt. Sie ist vollständig, wenn jede für $L$ stimmende Regel in dieser Weise gewonnen werden kann. Schließlich ist $\Theta$ für $L$ adäquat, wenn es für diese Sprache korrekt und vollständig ist. Nennen wir eine aus $\mathbf{K}(\Theta)$ mit Hilfe der Regeln des Regelwerks in endlich vielen (ggf. auch 0) Schritten gewinnbare semantische Regel ein Theorem von $\Theta$ und bezeichnen die Menge der Theoreme von $\Theta$ mit $\mathbf{T h}(\Theta)$, so gilt also, dass die Theorie $\Theta$ für L genau dann korrekt ist, wenn $\mathbf{T h}(\Theta)$ und $\mathbf{I n R}(\mathrm{L})$ disjunkte Klassen sind. $\Theta$ ist vollständig, wenn $\operatorname{KoR}(\mathrm{L})$ eine Teilklasse $\operatorname{von} \mathbf{T h}(\Theta)$ ist.

\section{Die Rolle von Bedeutungstheorien für die Semantik}

Nachdem wir uns nun gleichermaßen über Bedeutungstheorien und über Semantiken klar geworden sind, können wir die Beziehungen zwischen beiden diskutieren. Wir erklären im folgenden, welche Rolle bedeutungstheoretische Konzepte beim Aufbau semantischer Theorien spielen. Dabei verdeutlichen wir zunächst, wie eine semantische Theorie der Minimalsprache ML durch eine realistische Referenztheorie der Bedeutung fun- 
diert werden kann. Dies ist zum einen der einfachere Fall einer bedeutungstheoretischen Fundierung einer semantischen Theorie, zum anderen liefert dies eine Vergleichsfolie für den Fall der gebrauchstheoretischen Fundierung; wir greifen dies im 5. Abschnitt wieder auf.

\subsection{Bedeutungstheoretische Prinzipien in der Semantik}

In einer für $\mathrm{L}$ adäquaten Semantik $\Theta$ erhalten die nicht zum $\operatorname{Kern} \mathbf{K}(\Theta)$ gehörigen semantischen Regeln durch ihre Ableitung aus diesem Kern eine Erklärung. Insgesamt bewegt man sich aber mit $\Theta$ noch nicht einmal auf der Ebene der Beobachtungsadäquatheit, geschweige denn auf der Ebene der Erklärungsadäquatheit. Man hat mit $\Theta$ zwar eine adäquate Beschreibung der für $L$ einschlägigen semantischen Regeln, die über eine bloße Auflistung dieser Regeln hinausgeht. Aber es bleibet eine Reihe weitergehender Fragen offen:

- Wie lässt sich eigentlich die Beschränkung der Urteilsformen rechtfertigen, die zur genauen Abgrenzung der Klasse $\operatorname{ReR}(\mathrm{L})$ führt?

- $\operatorname{Th}(\Theta)$ kann Regeln enthalten, die nicht in $\mathbf{K o R}(L)$ (aber auch nicht in InR(L)) enthalten sind. Ich will solche Regeln als ideale Elemente von $\Theta$ bezeichnen ${ }^{14}$. Es kann sein, dass die Annahme solcher idealen Elemente für die deduktive Systematisierung der Regeln durch $\Theta$ unerlässlich sind. Wie aber ist die Annahme solcher idealen Elemente zu rechtfertigen?

- Offensichtlich beschränkt das gewählte Regelwerk die in einer Theorie ableitbaren Regeln. Welche speziellen Gründe motivieren dann aber die Wahl eines bestimmten Regelwerks?

Dies sind gerade Fragen, die jeweils im Rahmen von Bedeutungstheorien beantwortet werden. Bedeutungstheorien stellen also Prinzipien bereit, die die Form semantischer Theorien beschränken. Dies betrifft bereits die in diesen Theorien vorkommende Terminologie. Bekanntlich verwerfen einige Bedeutungstheorien bestimmte semantische Beziehungen als bloße „Scheinbegriffe“. So weist etwa Quine die Eigenschaften ,analytisch“ und „synthetisch“ sowie die Beziehung der (analytischen) Folge

14 Damit nehme ich eine Redeweise aus der Beweistheorie Hilberts auf. 
zurück; vgl. Quine (1960). In einer der Quineschen Bedeutungstheorie genügenden Semantik wären also nur Urteile der Form $\varphi:$ T und $\psi: F$, nicht aber Urteile der Form $\varphi<\psi$ zulässig.

Aber auch Erweiterungen des Regelwerks (12) (oder auch Abstriche an seinen Regeln) werden bedeutungstheoretisch gerechtfertigt. Zwei denkbare Zusätze wären etwa das Widerspruchsprinzip (Wid) und das Prinzip vom ausgeschlossenen Dritten (Tnd).

(13a)

(13b)

$$
\overline{\varphi: \mathrm{T}, \varphi: \mathrm{F} \Rightarrow\{\}}
$$

$$
\{\} \Rightarrow \varphi: \mathrm{T}, \varphi: \mathrm{F}
$$

(Wid)

Das Widerspruchsprinzip (13a) besagt, dass eine Aussage nicht zugleich wahr und falsch sein kann, während das Prinzip vom ausgeschlossenem Dritten behauptet, dass eine Aussage wahr oder falsch sein muss. Zusammen machen diese beiden Prinzipien das Bivalenzprinzip aus, demgemäss eine Aussage wahr oder falsch ist.

Im Gegensatz zu den drei Regeln des Regelwerks (12) sind die Prinzipien (Wid) und (Tnd) nicht strukturell, da sie ja Urteile bestimmter Formen betreffen. Sie sind aber auch nicht sprachbezogen, wie etwa die Regeln in (7), denn in seinen beiden Teilprinzipien ist ja von speziellen Zeichen der analysierten Sprache gar nicht die Rede. Solche Zeichen werden lediglich durch die griechischen Kleinbuchstaben schematisch angedeutet. Man könnte hier von ,semi-strukturellen“ Prinzipien reden.

Während das Widerspruchsprinzip im Rahmen der meisten Bedeutungstheorien akzeptiert wird, ist das Prinzip vom ausgeschlossenen Dritten umstritten. Für Dummett ist es ein Kennzeichen sog. ,realistischer Bedeutungstheorien“, s. Dummett (1993b: 468). Umgekehrt ist Ablehnung des Bivalenzprinzips ein Charakteristikum ,of the deepest and most interesting forms of antirealism“ (ebenda), zu denen etwa auch die als ,intuitionistisch“ bezeichnete Position in der Philosophie der Mathematik gehört. Typisch für realistische Bedeutungstheorien ist, dass sie die Bedeutsamkeit von Zeichen als unabhängig von den kognitiven Fähigkeiten der Zeichenbenutzer ansehen. Beim Bivalenzprinzip bedeutet dies, dass angenommen wird, eine Aussage aus dem Geltungsbereich dieses Prinzips 
habe einen bestimmten Wahrheitswert - wahr oder falsch - unabhängig von unseren Fähigkeiten, diesen auch zu erkennen ${ }^{15}$.

Eine Bedeutungstheorie erklärt, warum die von ihr angenommenen idealen Elemente als stimmend betrachtet werden können, obwohl über ihre Stimmigkeit empirisch nichts auszumachen ist, und sie erklärt weiter, warum die Regeln des gewählten Regelwerks von stimmenden semantischen Regeln wiederum zu solchen Regeln führen. Das Bivalenzprinzip erhält z. B. eine Rechtfertigung in einer wahrheitstheoretischen Bedeutungstheorie, wie sie Davidson entwickelt; vgl. die in Davidson (1984) gesammelten Aufsätze. Dieser Theorie zufolge gibt es zur analysierten Sprache $L$ eine Metasprache $M S$, in der die klassische zweiwertige Logik $^{16}$ gilt und sich ein Wahrheitsprädikat , $\in \mathrm{W}^{\text {“ }}$ in Übereinstimmung mit Tarskis Konvention W (s. Tarski 1936: 305f) unter Zuhilfenahme des Erfüllbarkeitsbegriffs definieren lässt. Übereinstimmung mit der Konvention $\mathrm{W}$ heißt, dass es zu einer jeden Aussage $\varphi$ von $L$ eine Übersetzung $A$ in $M S$ gibt, sodass in der Metasprache der Satz gilt, dass genau dann $\varphi \in \mathrm{W}$ ist, wenn $A$. Relativ zu einer solchen Definition ist dann eine Semantik $\Theta$ genau dann korrekt, wenn alle in $\Theta$ ableitbaren Regeln in richtige metasprachliche Aussagen übergehen, wenn man in den Regeln das Urteilsprädikat „, : T“ als Wahrheitsprädikat , $\in$ W“ liest; vgl. unten 5.2.

Zuhilfenahme der Erfüllbarkeitsrelation bedeutet, dass in $M S$ ein Bereich $U$ von Individuen zugrundegelegt wird, über die die Quantoren der Objektsprache $L$ laufen und auf die die Eigennamen dieser Sprache referieren. Die Prädikate der Sprache $L$ haben Teilmengen von $U$ als Extensionen, und in ähnlicher Weise beziehen sich sämtliche Kategore-

15 Intuitionistische und gebrauchstheoretische Vorstellungen gehen in den bedeutungstheoretischen Arbeiten von Dummett und Prawitz eine enge Verbindung miteinander ein; vgl. die Aufsatzsammlung Dummett (1993a) sowie Prawitz (1977 und 1982). Dass hier eine gewisse Affinität besteht, kann man auch daran ermessen, dass die Wiener Vorträge des niederländischen Mathematikers Brouwers, des Begründer des Intuitionismus, Wittgenstein 1928 dazu bewegt haben, sich nach längerer Pause wieder mit philosophischen Fragen zu beschäftigen; vgl. Schulte (1989: 18f, 118). Zu nicht-realistischen Bedeutungstheorien und darauf gründenden konstruktiven Semantikkonzepten vgl. Robering (1997: § 6).

16 In ihrer Version des Davidsonschen Programms geben Larson/ Segal (1995) die Annahme der vollen klassischen Logik in $M S$ auf und versuchen mit einer solchen Logik auszukommen, die gerade hinreichend ist, um alle W-Sätze - das sind die oben beschriebenen Sätze der Form $\varphi \in \mathrm{W} \leftrightarrow A-$ abzuleiten. 
mata von $L$ auf irgendwelche über $U$ gebildete mengentheoretische Konstrukte. Die Annahme eines solchen Individuenbereichs ist eine genuin bedeutungstheoretische, keine semantische Annahme. Die eigentliche Semantik von $L$ kommt ohne eine solche Annahme aus; sie ist in demselben Sinne „straight“, wie es für Leblanc die Logik in der von ihm präferierten Logikkonzeption ist:

"Some like their logic mixed with a lot of ontology. To them first-order logic, for example, adjudicates on basic matters of existence, and any semantic account of it should come with things, sets of things, and relations between things. [...] Others prefer their logic straight. [...] Of course, there are things, and if one is to discourse about them, he must - sooner or later - think of them as belonging to sets, bearing relations to one another, and so on. But first-order logic can be, and hence is perhaps best, explicated without recourse to 'models': What is dispensable simply is not of the essence" (Leblanc 1973: 241).

\subsection{Eine referenztheoretische Fundierung der Semantik von ML}

Wir nehmen nun wieder unsere Diskussion der Wittgensteinschen Minimalsprache ML auf; vgl. oben den Unterabschnitt 3.2. Als Regelkern unserer semantischen Theorie der Minimalsprache wählen wir die Regel (9) und die Instanzen des Schemas (10). Unser Regelwerk soll aus den drei Regeln von (12) und den beiden das Bivalenzprinzip bildenden Regeln von (13) bestehen.

Das folgende Beispiel zeigt, wie man aus dem Kern mit Hilfe des Regelwerks eine neue Regel ableiten kann. (Dabei sind triviale Anwendungen der Regel (M) fortgelassen worden.)

(14)

\{\}$\Rightarrow$ Würfel $: \mathrm{T}$, Säule $: \mathrm{T}$, Platte $: \mathrm{T}$, Balken $: \mathrm{T} \overline{\text { Würfel }: \mathrm{T}, \text { Würfel }: \mathrm{F} \Rightarrow\{\}}$

Würfel $: \mathrm{F} \Rightarrow$ Säule : T, Platte : T, Balken : T

Säule : $\mathrm{T}$, Säule $: \mathrm{F} \Rightarrow\{\}$

Würfel : F, Säule : F $\Rightarrow$ Balken : T, Platte $: \mathrm{T}$

Eine Erklärung unserer semantischen Theorie im Rahmen einer wahrheits- und referenztheoretischen Bedeutungstheorie, wie sie Davidson vertritt, würde nun so aussehen: Wir geben für unsere Minimalsprache eine prädikatenlogische Metasprache an, deren Variablen über die Bauelemente laufen, mit denen der Bauende und der Gehilfe umgehen. In der Metasprache gibt es ferner die Individuenkonstanten Platte, Würfel, Säule 
und Balken für die vier Ausdrücke von ML. Ferner haben wir in der Metasprache die vier Prädikate Cube, Column, Slab und Beam.

Den Ausdruck Würfel der Minimalsprache übersetzen wir naheliegenderweise in die Formel Cube $(x)$ der Metasprache. Die freie Variable ,,$x^{“}$ kann dabei je nach Verwendungskontext des Ausdrucks unterschiedliche Werte annehmen. Sie modelliert also das deiktische Moment der Minimalsprache. Entsprechende Übersetzungen wie für Würfel gibt es natürlich auch für die drei anderen Ausdrücke der Minimalsprache.

Die Erfüllbarkeitskeitsrelation ist eine zweistellige Beziehung zwischen Objekten und Ausdrücken der Minimalsprache: Wir kürzen sie, um den Zusammenhang mit unserer semantischen Theorie klar hervortreten zu lassen, durch $\varphi \in \mathrm{W}_{x}$ ab: Objekt $x$ erfüllt den Ausdruck $\varphi$ (oder auch: $\varphi$ ist wahr bei $x$ ). Wegen der Endlichkeit der Minimalsprache lässt sich das Erfüllbarkeitsprädikat sogar ohne Rekursion definieren.

$$
\begin{aligned}
& \varphi \in \mathrm{W}_{x} \leftrightarrow[\varphi=\text { Würfel } \wedge \text { Cubus }(\mathrm{x})] \\
& \\
& \vee[\varphi=\text { Säule } \wedge \text { Column }(\mathrm{x})] \\
& \vee[\varphi=\text { Balken } \wedge \operatorname{Beam}(\mathrm{x})] \\
& \vee[\varphi=\text { Platte } \wedge \operatorname{Slab}(\mathrm{x})]
\end{aligned}
$$

Urteile der Form $\varphi$ : T erhalten nun die durch $x$ parametrisierten Übersetzungen der Form $\varphi \in \mathrm{W}_{x}$ zugewiesen. Negative Urteile $\varphi$ : F interpretieren wir entsprechend durch metasprachliche Formeln der Gestalt $\neg(\varphi \in$ $\mathrm{W}_{x}$ ). Innerhalb der wahrheits- und referenztheoretischen Bedeutungstheorie wird dann weiter eine semantische Regel

$$
\begin{aligned}
& \varphi_{1}: \mathrm{T}, \ldots, \varphi_{m}: \mathrm{T}, \varphi_{m+1}: \mathrm{F}, \ldots, \varphi_{m+n}: \mathrm{F} \Rightarrow \psi_{1}: \mathrm{T}, \ldots, \psi_{p}: \mathrm{T}, \psi_{p+1}: \\
& \mathrm{F}, \ldots, \psi_{p+r}: \mathrm{F}
\end{aligned}
$$

durch die generalisierte Implikation

$$
\begin{aligned}
& x\left[\varphi_{1} \in \mathrm{W}_{x} \wedge \ldots \wedge \varphi_{m} \in \mathrm{W}_{x} \wedge \neg\left(\varphi_{m+1} \in \mathrm{W}_{x}\right) \wedge \ldots \wedge \neg\left(\varphi_{m+n} \in \mathrm{W}_{x}\right)\right. \\
\rightarrow & \left.\psi_{1} \in \mathrm{W}_{x} \vee \ldots \vee \Psi_{p} \in \mathrm{W}_{x} \vee \neg\left(\Psi_{p+1} \in \mathrm{Wx}\right) \vee \ldots \vee \neg\left(\psi_{p+r} \in \mathrm{W}_{x}\right)\right]
\end{aligned}
$$

gedeutet ${ }^{17}$.

Bei dieser Art der Deutung besagt die semantische Regel (9), dass der Gegenstandsbereich $U$ die Vereinigung von vier den Ausdrücken Würfel,

17 Ist dabei $m+n=0$, also das Antezedens der zu übersetzenden Regel leer, so wählen wir als Vorderglied der Implikation eine beliebige tautologische Formel. Entsprechend wird bei leerem Sukzedens, also im Falle $p+r=0$, ein kontradiktorisches Hinterglied gewählt. 
Säule, Platte und Balken entsprechenden Klassen ist. Die Regel (10) besagt, dass diese Klassen disjunkt sind. Beide Regel zusammen drücken also aus, dass $U$ in vier disjunkte Klassen zerlegt wird, die den Ausdrücken der Minimalsprache korrespondieren. Mit dem Widerspruchsprinzip (Wid) wird gesagt, dass kein Objekt von $U$ einer der vier Klassen angehören und zugleich außerhalb derselben Klasse liegen kann. Das Prinzip (Tnd) vom ausgeschlossenen Dritten behauptet, dass ein jedes Objekt aus $U$ zu einer Klasse gehört oder nicht gehört - ganz unabhängig davon, ob wir oder ein Teilnehmer an $\mathbf{W}$ dies auch feststellen können.

\section{Gebrauchstheoretisch fundierte Semantik}

Im Unterabschnitt 4.2 ist kurz skizziert worden, wie eine Fundierung einer semantischen Theorie für ML durch eine Wahrheits- und Referenztheorie der Bedeutung aussehen kann. Jetzt soll der Frage nachgegangen werden, wie eine gebrauchstheoretische Fundierung der Semantik aussehen könnte. Ich sehe dabei von der Verflechtung unterschiedlicher Sprachspiele in einer Lebensform (s. o. 2.4) ab und gehe von der vereinfachenden Hypothese aus, dass einer Sprache $L$ nur ein einziges Sprachspiel $\boldsymbol{G}$ entspricht, das in einem explanativen Zusammenhang mit der semantischen Theorie $\Theta$ von $L$ gebracht werden muss.

\subsection{Die technische Form des Problems}

Einen Teilaspekt des Problems können wir in abstrakter Weise folgendermaßen charakterisieren: Die Partien von $\boldsymbol{G}$ sind endliche nichtleere Folgen von Äußerungs- und Nicht-Äußerungszügen, also von Elementen von $\mathbf{U Z}(\boldsymbol{G})$ und von $\mathbf{N Z}(\boldsymbol{G})$. Wir können also die Menge der Partien im Sinne der mathematischen Linguistik als eine Sprache $\mathbf{L}(\boldsymbol{G})$ über dem Alphabet $\mathbf{V}(G):=\mathbf{U Z}(G) \cup \mathbf{N Z}(G)$ ansehen: $\mathbf{L}(G) \subseteq(\mathbf{U Z}(G) \cup \mathbf{N Z}(G))^{+}$; vgl. etwa Aho/ Ullman (1972) ${ }^{18}$. Die Frage, ob die Gebrauchstheorie der Bedeutung in einer semantischen Theorie $\Theta$ zu gebrauchen ist, lässt sich dann auf einer technischen Ebene so stellen: Gibt es einen Zusammenhang zwischen der Sprache $\mathbf{L}(\boldsymbol{G})$ und der Regelmenge $\mathbf{T h}(\Theta)$ ?

18 In mehreren Arbeiten aus den 70er Jahren hat Maria Nowakowska die Idee entwickelt, Konzepte und Methoden der mathematischen Linguistik auf die Analyse von Handlungszusammenhängen anzuwenden; vgl. Nowakowska (1973 und 1980). 
Idealerweise sollte dieser Zusammenhang zwischen $\mathbf{L}(\boldsymbol{G})$ und $\mathbf{T h}(\Theta)$ algorithmischer Natur und in einem zu präzisierenden Sinne effektiv lernbar sein. $\mathbf{T h}(\Theta)$ stellt ja die semantische Kompetenz eines Mitgliedes der Sprachgemeinschaft dar, und diese kann nur durch aktive Teilnahme an dem Sprachspiel $\boldsymbol{G}$ gewonnen worden sein; vgl. den Punkt (4) in der Auflistung von 2.1. Ein Sprecher mit seinen begrenzten Berechnungsfähigkeiten muss also zunächst von seinem beobachteten endlichen Ausschnitt aus $\mathbf{L}(\boldsymbol{G})$ auf $\mathbf{T h}(\Theta)$ schließen.

\subsection{W-Spiel und ML in gebrauchstheoretischer Sicht}

In unserem Beispiel können wir einen Zusammenhang der beschriebenen Art wie folgt herstellen: Die Äußerungen im Sprachspiel sind Befehle, die Reaktionshandlungen in Partien des Spiels stellen jeweils einen Zustand her, den der jeweils vorangehende Befehl als erwünscht präsentiert. In einer Partie dieses Spiels präsentiert also ein Befehl eine Zustandseigenschaft, die der durch die nachfolgende Reaktionshandlung herbeigeführte Zustand gerade aufweist.

Die grammatische Struktur der Sprache $\mathbf{L}(\mathbf{W})$ des $\mathbf{W}$-Spiels ist also denkbar einfach. Ihr Vokabular besteht aus acht Symbolen, die man, wenn man von der internen Struktur der Züge absieht, einfach durch $\alpha_{1}, \alpha_{2}, \beta_{1}$, $\beta_{2}, \gamma_{1}, \gamma_{2}, \delta_{1}$ und $\delta_{2}$ darstellen kann, wobei das mit „1“ indizierte Symbol jeweils einen der vier möglichen Äußerungszüge und das entsprechende mit ,2“ indizierte Symbol den zugehörigen Reaktionszug repräsentieren soll. Die Sprache $\mathbf{L}(\mathbf{W})$ wird dann durch den regulären Ausdruck $\left(\alpha_{1} \alpha_{2}\left|\beta_{1} \beta_{2}\right| \gamma_{1} \gamma_{2} \mid \delta_{1} \delta_{2}\right)\left(\alpha_{1} \alpha_{2}\left|\beta_{1} \beta_{2}\right| \gamma_{1} \gamma_{2} \mid \delta_{1} \delta_{2}\right)^{*}$ beschrieben ${ }^{19}$, ist also selbst regulär; vgl. Aho/ Ullman (1972: ch. 2.2).

Die Sprache $\mathbf{L}(\mathbf{W})$ hat einen ausgesprochen ,lokalen“ Charakter: Die einzig interessanten grammatischen Abhängigkeiten zwischen den Symbolen ihrer Wörter bestehen zwischen jeweils unmittelbar aufeinanderfolgenden Symbolen: zwischen einem Äußerungszug und dem sofort folgenden Reaktionszug. Diese Beziehung ist für die Semantik der im W-Spiel benutzten Sprache ML aber von fundamentaler Wichtigkeit.

19 Der oben angegebene reguläre Ausdruck besagt, dass ein Wort von $\mathbf{L}(\mathbf{W})$ eine zweisymbolige Folge von Äußerungs- und zugehörigem Reaktionszug ist, an die sich noch weitere solcher Abfolgen anschließen können (aber nicht müssen). 
Man könnte in der Sprache einer realistischen Bedeutungstheorie sagen, der Reaktionszug verifiziere den Äußerungszug. Ganz ohne von einer solchen realistischen Ausdrucksweise Gebrauch zu machen, können wir diese Beziehung auch in der folgenden Weise für die Deutung von semantischen Urteilen heranziehen:

(18) Für eine Tätigkeit $t$ ist genau dann $\varphi \in V_{t}$, wenn es ein Wort $w=$ $z_{1} \ldots z_{m} z_{m+1} \ldots z_{n} \in \mathbf{L}(\mathbf{W})$ (also eine korrekte Partie unseres Sprachspiels) mit den folgenden Eigenschaften gibt:

a) $z_{m}$ hat die Form (Bauender, $\varphi$, !, Gehilfe).

b) $z_{m+1}$ hat die Form (Gehilfe, $t$ ).

Die so definierte Beziehung zwischen Ausdrücken $(\varphi)$ und Tätigkeiten $t$ übernimmt im gebrauchstheoretischen Rahmen die Rolle, die in der realistischen Referenztheorie die Erfüllbarkeitsbeziehung zwischen Ausdrücken und Gegenständen spielt.

Die Regel (16) können wir nun in zu (17) analoger Weise interpretieren, wodurch wir eine gebrauchstheoretische Fundierung der semantischen Theorie der Minimalsprache erhalten. Hierbei spielt die in (18) eingeführte gebrauchstheoretische Bedingung $\varphi \in \mathrm{V}_{t}$ die Rolle des referenztheoretischen $\varphi: \mathrm{W}_{x}$. Die semantische Regel (9) der Minimalsprache ist dann ein Reflex des Umstandes, dass jede Reaktionshandlung des Gehilfen adäquate Reaktion auf eine der vier möglichen Befehle des Bauenden ist; und die Regel (10) spiegelt wider, dass keine Tätigkeit des Gehilfen für mehr als nur einen Befehl adäquate Reaktion ist. Das Widerspruchsprinzip (Wid) beinhaltet, dass eine Tätigkeit des Gehilfen nicht zugleich eine adäquate und eine inadäquate Reaktion auf eine Äußerung des Bauenden sein kann. (Tnd) besagt, dass ein Reaktionszug stets adäquat oder inadäquat ist.

\subsection{Eine Erweiterung des W-Spiels}

Die gebrauchstheoretische Fundierung der Semantik der Minimalsprache von 5.2 unterscheidet sich nicht wesentlich von der referenztheoretischen in 4.2. Dies liegt aber lediglich an der Einfachheit des W-Spiels. In ihm hat z. B. der Gehilfe keine Möglichkeit, eine Aufforderung des Bauenden abzulehnen. Räumen wir ihm in einer Erweiterung W' des W-Spiels diese Möglichkeit ein, indem wir etwa das Arsenal der Tätigkeiten durch eine Aktion \& (etwa ein Handzeichen) erweitern, mit der er eine Aufforderung 
des Bauenden ablehnen und eine Partie des W-Spiels beenden kann, so ergeben sich bei der gebrauchstheoretischen Deutung des „: : F“-Prädikats der Minimalsprachensemantik neue Möglichkeiten.

Man beachte, dass es sich bei der Sprache von W' nach wie vor um die Minimalsprache handelt, die auch in $\mathbf{W}$ benutzt wird. Die referenztheoretisch fundierte Semantik bleibt von der Erweiterung von W zu W' auch völlig unberührt: Durch die Möglichkeit, Aufforderungen abzulehnen, ändert sich ja nichts an der Zerlegung des Gegenstandsbereichs $U$ in vier disjunkte Klassen. Im gebrauchstheoretischen Ansatz können wir zwar (18) ebenfalls einfach übernehmen, da ja in $\mathbf{W}^{\text {' * eine }}$ - im Sinne der Grammatik von $\mathbf{L}\left(\mathbf{G}^{\prime}\right)$ - adäquate Reaktion auf jeden Äußerungszug des Bauenden ist. Es muss aber (18) noch um die Bestimmung (19) einer Beziehung $Z$ des Zurückweisens ergänzt werden, die * als einzige Zurückweisung einer jeden Aufforderung charakterisiert.

(19) Es ist $\varphi \in Z_{\star}$ für jeden Ausdruck $\varphi$ der Minimalsprache, und aus $\varphi \in Z_{t}$ folgt $t=*$.

Ein negatives Urteil $\varphi:$ F wird nun nicht einfach in Analogie zu 4.2 durch die Negation $\neg\left(\varphi \in \mathrm{V}_{t}\right)$ interpretiert, sondern durch $\varphi \in Z_{t}$. Bei dieser Interpretation der Semantik bleiben die Regeln (9) und (10) für ML, die gewissermaßen empirische Generalisierungen dieser Sprache darstellen, korrekt. Die beiden in 4.1 als ideale Elemente bezeichneten Regeln (Wid) und (Tnd), die zusammen das Bivalenzprinzip ausmachen, gelten aber nicht mehr $^{20}$.

Man sieht also, dass hier ein gebrauchstheoretischer Ansatz zu einer ganz anderen Semantik der Minimalsprache führen würde als ein referenztheoretischer.

\subsection{Ein gebrauchstheoretisches Programm}

Ob die Frage nach der Brauchbarkeit der Gebrauchstheorie der Bedeutung in der Semantik einer vorgegebenen Sprache $L$ positiv zu beantworten ist, hängt insbesondere von der Durchführbarkeit des folgenden Programms ab:

\footnotetext{
20 Man könnte dieses Beispiel auch durch Modifikation der Übersetzungsvorschrift, die semantische Urteile in metasprachliche Formeln überführt, so variieren, dass sich (Wid) als gültig und (Tnd) als ungültig herausstellt. Man hat lediglich als Übersetzung eines leeren Sukzedens eine Bedingung zu wählen, die nur von \& erfüllt wird.
} 
1 Es ist ein für die Sprachgemeinschaft von $L$ erschöpfender Katalog der illokutionären Kräfte zu erstellen, mit denen die Mitglieder der Sprachgemeinschaft die Ausdrücke von $L$ äußern.

2 Es ist für diese Sprachgemeinschaft eine vollständige und explizite Charakterisierung der in ihr üblichen Sprachspiele anzugeben.

Die Notwendigkeit des ersten Programmpunktes geht aus unserem einfachen Beispiel hervor. Die vorgeführte gebrauchstheoretische Interpretation der semantischen Regeln der Minimalsprache machte wesentlichen Gebrauch davon, dass die Tätigkeiten des Gehilfen Reaktionen auf Befehle des Bauenden sind. Und es ist schwer einzusehen, wie syntaktische Muster, die sich in der ,Zugsprache“ der unterschiedlichen Sprachspiele zeigen, ohne die Information darüber zu deuten sind, wie ihre einzelnen Züge gemeint sind.

Was den zweiten Programmpunkt anbelangt, so hat man natürlich die Möglichkeit einzuräumen, dass nicht alle Aspekte des Gebrauchs sprachlicher Einheiten semantisch relevant sein müssen. Ein Ausdruck einer natürlichen Sprache wird in einer Vielzahl von Sprachspielen vorkommen; aber nicht jedes dieser Sprachspiele muss die Bedeutung des Ausdrucks affizieren. Es wäre denkbar, dass es unter den ,unzähligen(n)“ (Wittgenstein 1958/69: 300) Sprachspielen eine kleine Klasse von Standardspielen gibt, die allein von semantischer Relevanz sind. Um dies feststellen zu können, müsste man vorab aber einen Überblick über den gesamten Gebrauch der Sprache haben.

Der konstruktive Aufbau einer gebrauchstheoretisch fundierten Semantik wird, wenn ein solcher überhaupt möglich ist, also von einem möglichst kompletten Überblick über die Gesamtheit der Sprachspiele ausgehen. Umgekehrt ist die Unmöglichkeit einer gebrauchstheoretisch fundierten Semantik erst dann erwiesen, wenn man zeigt, dass die semantischen Regeln selbst dann nicht gebrauchstheoretisch erklärt werden können, wenn man alle regelhaften Aspekte des Sprachgebrauchs, also alle Sprachspiele berücksichtigt. 


\section{Literatur}

Aho, Alfred V. \& Ullman, Jeffrey D. (1972). The theory of parsing, translation and compiling. Vol. 1. Parsing. Englewood Cliffs NJ: Prentice Hall.

Alston, William (1964). Philosophy of Language. Englewood Cliffs NJ: Prentice Hall.

Balzer, Wolfgang, Moulines, C. Ulises \& Sneed, Joseph D. (1987). An architectonic for science. The structuralist program. Dordrecht: Reidel.

Bloomfield, Leonard (1926). A set of postulates for the science of language. In Language 2. 153-164.

Davidson, Donald (1984). Inquiries into truth and interpretation. Oxford: Clarendon Press.

Dummett, Michael (1991a). Frege and other philosophers. Oxford: Clarendon Press.

Dummett, Michael (1991b). Frege and Wittgenstein. In Dummett (1991a). 237-248.

Dummett, Michael (1993a). The seas of language. Oxford: Oxford University Press.

Dummett, Michael (1993b). Realism and anti-realism. In Dummett (1993a). 462-478.

Gentzen, Gerhard (1934/1935). Untersuchungen über das logische Schließen. In Mathematische Zeitschrift 39. 176-210 und 405-431.

Hallett, Garth (1967). Wittgenstein's definition of meaning as use. New York: Fordham University Press.

Hamblin, Charles Leonard (1971). Mathematical models of dialogue. In Theoria 37. $130-155$.

Hintikka, Jaakko (1973). Logic, Language-Games and Information. Kantian themes in the philosophy of logic. Oxford: Clarendon Press.

Hintikka, Jaakko (1996). Ludwig Wittgenstein: half-truths and one-and-a-half-truths. Dordrecht: Kluwer.

Hintikka, Jaakko (2000). On Wittgenstein. Belmont CA: Wadsworth.

Hintikka, Jaakko \& Kulas, Jack (1983). The game of language. Studies in game-theoretical semantics and its applications. Dordrecht: Reidel.

Kecskeméti, Paul (1952). Meaning, communication and value. Chicago: University of Chicago Press.

Larson, Richard \& Segal, Gabriel (1995). Knowledge of meaning. An introduction to semantic theory. Cambridge MA: MIT Press.

Leblanc, Huges (1973). On dispensing with things and worlds. In Munitz, Milton K. (ed.) (1973). Logic and ontology. New York: New York University Press. 241-259.

Leisi, Ernst (1952). Der Wortinhalt. Seine Struktur im Deutschen und Englischen. Heidelberg: Quelle und Meyer. 5. Auflage 1975.

Lenk, Hans (Hg.) (1980). Handlungstheorien - interdisziplinär. Bd. I. Handlungslogik, formale und sprachwissenschaftliche Handlungstheorien. München: Fink. 
Lorenzen, Paul \& Lorenz, Kuno (ed.) (1978). Dialogische Logik. Darmstadt: Wissenschaftliche Buchgesellschaft.

Lyons, John (1977). Semantics. Vol. 1. Cambridge: Cambridge University Press.

Margalit, Avishai (ed.) (1979). Meaning and use. Papers presented at the Second Jerusalem Philosophical Encounter April 1976. Dordrecht: Reidel and Jerusalem: Magnes Press.

Nowakowska, Maria (1973). Language of motivation and language of action. Paris, den Haag: Mouton.

Nowakowska, Maria (1980). Eine formale Theorie der Handlungen. In Lenk (1980). 167-221.

Posner, Roland, Robering, Klaus \& Sebeok, Thomas A. (Hgg.) (1997). Semiotik. Ein Handbuch zu den zeichentheoretischen Grundlagen von Natur und Kultur. Bd. I. Berlin, New York: de Gruyter.

Prawitz, Dag (1977). Meaning and proofs: on the conflict between classical and intuitionistic logic. In Theoria 43. 2-40.

Prawitz, Dag (1982). Beweise und die Bedeutung und Vollständigkeit der logischen Konstanten. In Conceptus 16. 31-44.

Quine, Willard V. (1960). Word and object. Cambridge MA: MIT Press.

Quine, Willard V. \& Ullian, Joseph S. (1970). The Web of belief. New York: Random House.

Robering, Klaus (Hg.) (1992a). Wegweiser zur Bedeutung. Berlin: Technische Universität Berlin (= Arbeitspapiere zur Linguistik - Working papers in linguistics. 28).

Robering, Klaus (1992b). Werden Bedeutungen von Gebrauchsbedingungen festgelegt? In Robering (1992a). 61-98.

Robering, Klaus (1997). Semantik. In Posner/ Robering/ Sebeok (1997). 83-219.

Rückert, Helge (2001). Why dialogical logic? In Wansing (2001). 165-185.

de Saussure, Ferdinand (1967). Grundfragen der allgemeinen Sprachwissenschaft. Berlin: de Gruyter. 2. Auflage.

von Savigny, Eike (1994). Wittgensteins „Philosophische Untersuchungen“. Ein Kommentar für Leser. Frankfurt a. M.: Klostermann. 2. Auflage.

Schulte, Joachim (1989). Wittgenstein. Eine Einführung. Stuttgart: Reclam.

Scott, Dana (1971). On engendering an illusion of understanding. In Journal of philosophy 68. 787-802.

Tarski, Alfred (1936). Der Wahrheitsbegriff in den formalisierten Sprachen. In Studia Philosophica Commentarii Societatis philosphicae Polonorum 1. 261-405.

Tennant, Neil (1987). Anti-realism and logic. Truth as eternal. Oxford: Clarendon Press.

Wansing, Heinrich (ed.) (2001). Essays on non-classical logic. Singapur etc.: World Scientific. 


\section{6}

Wiegand, Herbert Ernst (Hg.) (1999a). Sprache und Sprachen in den Wissenschaften. Geschichte und Gegenwart. Festschrift für Walter de Gruyter \& Co. anlässlich einer 250jährigen Verlagstradition. Berlin: de Gruyter.

Wiegand, Herbert Ernst (1999b). Mit Wittgenstein über die Wortbedeutung nachdenken. Gebrauch? Regel des Gebrauchs? Ein Etwas im Kopf? In Wiegand (1999a). 404461.

Wittgenstein, Ludwig (1958). Philosophical investigations - Philosophische Untersuchungen. Oxford: Blackwell. - Wieder in Schriften. Bd. 1. Frankfurt a. M.: Suhrkamp 1969. 279-544. 